\title{
Machine integrated telecentric surface metrology in laser structuring systems
}

\author{
Robert Schmitt $^{12}$, Tilo Pfeifer ${ }^{12}$, Guilherme Mallmann ${ }^{2}$ \\ ${ }^{1}$ Laboratory for Machine Tools and Production Engineering WZL, RWTH Aachen University, Aachen, Germany \\ ${ }^{2}$ Fraunhofer Institute for Production Technology (IPT) - Department of Production Metrology, Aachen, Germany
}

\begin{abstract}
The laser structuring is an innovative technology used in a broad spectrum of industrial branches. There is, however, a market trend to smaller and more accurate micro structures, which demands a higher level of precision and efficiency in this process. In this terms, an inline inspection is necessary, in order to improve the process through a closed-loop control and early defect detection. Within this paper an optical measurement system for inline inspection of micro and macro surface structures is described. Measurements on standards and laser structured surfaces are presented, which underline the potential of this technique for inline surface inspection of laser structured surfaces.
\end{abstract}

\section{Section: RESEARCH PAPER}

Keywords: inline metrology, frequency-domain optical coherence tomography, surface inspection, laser structuring

Citation: Robert Schmitt, Tilo Pfeifer, Guilherme Mallmann, Machine integrated telecentric surface metrology in laser structuring systems, Acta IMEKO, vol. 2, no. 2, article 13, December 2013, identifier: IMEKO-ACTA-02 (2013)-02-13

Editor: Paolo Carbone, University of Perugia

Received April $15^{\text {th }}, 2013$; In final form October $8^{\text {th }}, 2013$; Published December 2013

Copyright: (c) 2013 IMEKO. This is an open-access article distributed under the terms of the Creative Commons Attribution 3.0 License, which permits unrestricted use, distribution, and reproduction in any medium, provided the original author and source are credited

Funding: This work was supported by German Federal Ministry of Education and Research (BMBF), Germany

Corresponding author: Guilherme Mallmann, e-mail: Guilherme.mallmann@ipt.fraunhofer.de

\section{INTRODUCTION}

The functionalization of surfaces based on laser micromachining is an innovative technology used in a broad spectrum of industrial branches. Its main advantage against other processes is the high machining flexibility. This technique permits the structuring of different workpieces (different form complexity and materials) with the same machine tool. Examples can be found in minimizing air resistance, operation noise and friction losses [1] as well as in the surface structuring of tools and moulds [2].

There is, however, a trend towards the improvement of the overall process quality and automation as well as smaller microstructures in laser micro-machining. The fulfilment of these demands are at present limited by the non-existence of a sufficiently described process model as well as the absence of a robust and accurate inline process monitoring technique.

On the one hand the missing process model leads to a time consuming effort to initialize the laser structuring of new products. This procedure depends e.g. on the applied material composition, product form and surface roughness. If the process behaviour is unknown for a determined workpiece, laser parameters and suitable machining strategies have to be identified in a trial and error testing before the real machining process can start. In this context reference geometries need to be structured and analysed outside the machine tool until a suitable parameter set is found. On the other hand the absence of a process control based on the real machined surface causes a high degree of inefficiency. This is explained by the inability of the machine to identify process defects during the machining procedure, leading to an increased possibility of rejected parts with a high degree of added value.

For solving this task with a high level of compatibility and integration, an optical distance measurement system based on the frequency domain optical coherence tomography (FD-OCT) was developed. The described telecentric measurement through the laser machining optical system enables a fast and highly accurate surface inspection in machine coordinates before, during and after the structuring process. Based on this process monitoring a machining control can be set-up, leading to a fully automated process adjustment and manufacture procedure. 


\section{STATE OF THE ART}

\subsection{Laser structuring process}

The laser structuring technology utilizes thermal mechanisms to machine a workpiece, which are induced through the absorption of high amounts of energy. The physical interaction of laser radiation and matter is therefore a crucial point in this process. Its efficiency is associated with laser and material properties, as applied wavelength, focal radius, angle of incidence, material light absorption, surface roughness, metal temperature, laser pulse repetition frequency and energy [1]. Typical working wavelengths for the machining of metals and their alloys are $1064 \mathrm{~nm}$ (infra-red light) or 532 $\mathrm{nm}$ (green light). The guidance of the laser beam to the part's surface is accomplished in most systems using a rastering system or a laser scanner [3]. This configuration uses computer numerically controlled galvanometer mirrors to deflect and an $\mathrm{f}$-theta lens to focus the laser beam over a working area. This lens is wavelength optimized to focus the chief ray of the laser beam normal to the scanning field regardless of the scan angle, as well as to make the traveled distance of the laser spot on the focal plan directly proportional to the scan angle [4].

\subsection{Laser structuring process monitoring}

Inline process monitoring solutions for laser based structuring systems currently being developed in the academy and industry show technical limitations. The technologies available present in part low accuracy, no depth information or are not able to measure directly in machine coordinates.

For example an approach using conoscopic holography is used. This technique is not able to measure directly in machine coordinates, as it uses a different optical path as the process laser, leading to complex calibration steps and inserting transformation errors as well as measurement displacement.

In [5] a technology based on the acquisition of process generated electro-magnetic emissions is presented for the monitoring of the selective laser melting process. A similar approach can be also applied on laser structuring systems. This technique is however not able to deliver any direct depth information, just being able to monitor the amount of energy absorbed in the machining procedure and based on this evaluate the removed depth.

\section{SOLUTION CONCEPT}

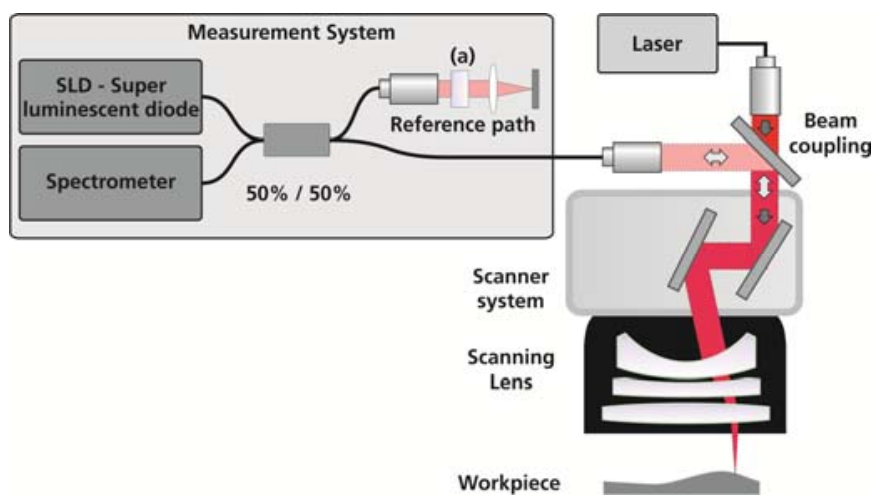

Figure 1. Concept of the inline process monitoring system - (a) dispersion compensator / glass rod.
The solution concept for the machine integrated process monitoring system was designed based on the rastering system machine layout (Figure 1). As measurement system an optical distance measurement technique based on the frequency domain optical coherence tomography (FD-OCT) was used. The system integration is accomplished through an optical element as beam splitter.

\subsection{Frequency domain - optical coherence tomography (FD-OCT)}

The FD-OCT is a technique based on low-coherence interferometry. Differently from normal low-coherence interferometers, which use a piezo element to find the maximum interference point, in the FD-OCT the depth information is gained by analyzing the spectrum of the acquired interferogram. The calculation of the Fourier transformation of the acquired spectrum provides a back reflection profile as a function of the depth. For the generation of the interference pattern a measurement and a reference path are used, where the optical path difference between these arms is detected. The higher the optical path difference between reference and measuring arm, the higher the resulting interference modulation (Figure 2).

The total interference signal $I(k)$ is given by the spectral intensity distribution of the light source $(G(k))$ times the square of the sum of the two back reflected signals $\left(a_{R}\right.$ as the reflection amplitude of the coefficient reference arm and $a(z)$ as the backscattering coefficient of the object, with regard to the offset $\left.z_{0}\right)$, where $k$ is the optical wavenumber [6]:

$$
I(k)=G(k)\left|a_{R} \exp (i 2 k r)+\int_{z_{0}}^{\infty} a(z) \exp \{i 2 k n(z)(r+z) d z\}\right|
$$

where $n$ is the refractive index, $2 r$ is the path length in the reference arm, $2(r+z)$ is the path length in the object arm and $2 z$ the difference in path length between both arms. By finding the maximum amplitude at the spectrum's Fourier transformation, the absolute optical path difference can be detected. The max. measuring depth $\left(Z_{\max }\right)$ is described by [7]

$Z_{\max }=\left(\lambda_{0}^{2} / 4 n \Delta \lambda\right) N$

where $\lambda_{0}$ is the central wavelength, $\Delta \lambda$ is the bandwidth, $n$ is the sample's refractive index and $N$ is the number of detector units covered by the light source's spectrum.

The axial resolution of an FD-OCT is described by [7]

$A R_{F D-O C T}=l_{c} / 2 \approx 0.44 \lambda_{0}^{2} / \Delta \lambda$.

For the measurement of single distance (a single back reflection) the axial resolution can be increased to a submicrometric resolution by the usage of signal processing techniques, such as gauss fit.

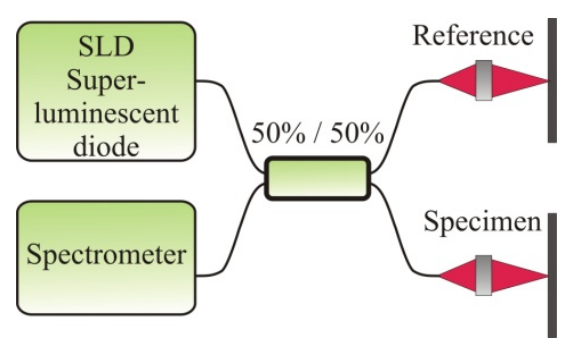

Figure 2. Frequency domain optical coherence tomography (FT-OCT) Set-up. 


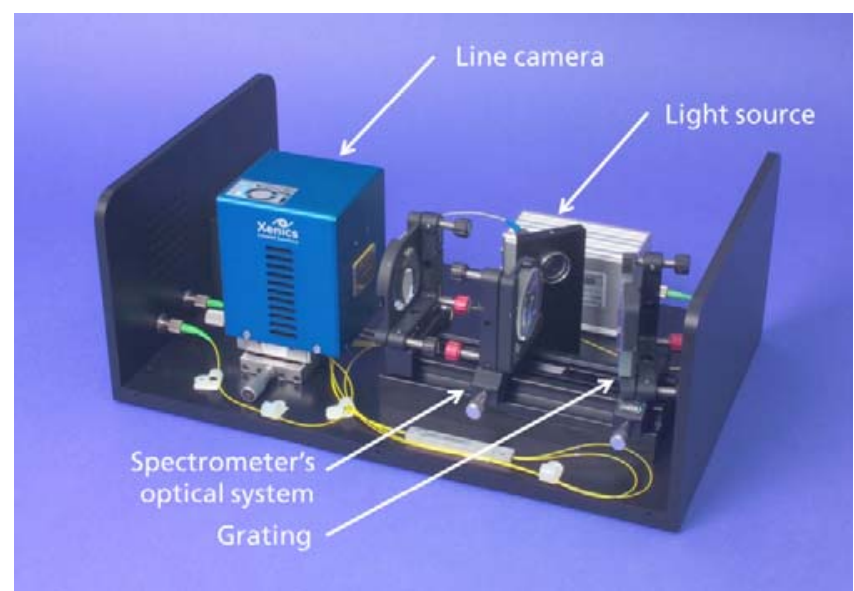

Figure 3. Measurement system based on Fourier domain low coherence tomography.

\subsection{Measurement system prototype}

The spectrometer for the interference signal acquisition was developed with a wavelength measuring range of $107 \mathrm{~nm}$, which can be adjusted in the absolute frequency range from 900 to $1100 \mathrm{~nm}$ depending on the light source to be used (Figure 3). As a detector an indium gallium arsenide (InGaAs) line camera was used. Standard silicon based detectors present quantum efficiencies of less than $20 \%$ for the applied wavelength range against values between $60 \%-80 \%$ for InGaAs $(1.7 \mu \mathrm{m})$ based detectors [8]. The light source used in the system is a superluminescent diode with central wavelength at $1017 \mathrm{~nm}$ and wavelength range of $101 \mathrm{~nm}$.

The theoretical measuring range (maximum depth scan) of the developed FD-OCT using the presented SLD light source was calculated using Equation (2) to be of $1.31 \mathrm{~mm}$. The available measurement range was evaluated using a precision linear translation table. The results showed a maximum distance measurement of $1.25 \mathrm{~mm}$ using a specimen of aluminium with a technical surface, which simulates the workpieces used for the laser structuring. The theoretical axial resolution of the system calculated based on Equation (3) is $4.51 \mu \mathrm{m}$. The usage of a gauss fitting algorithm to find the modulation frequency after the Fourier transformation of the acquired light spectrum increases the axial resolution by calculating a sub-pixel accurate curve maximum. Based on this technique an increased axial resolution could be achieved. The standard deviation of the distance measurement values acquired in the centre of the scanner field was $218 \mathrm{~nm}$. A detailed analysis of the measurement system is presented in [9].

\subsection{Beam coupling}

The concept chosen for the integration of the developed FD-OCT in a laser structuring machine is based on an optical filter or a dichroic beam splitter used as an optical coupler. The coupling is accomplished by using this optical element in the reflective area for the measurement wavelengths and in the transmissive area for the structuring laser wavelength.

A very important requirement on this system is the laser beam's transmission efficiency. The laser beam coupling performance will be directly connected to the machine process energy efficiency as well as to the overall heat development on

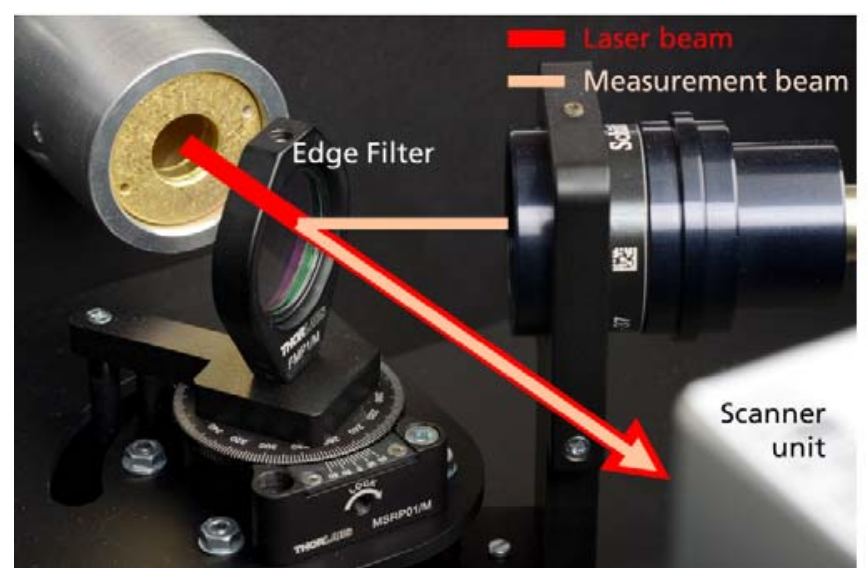

Figure 4. System prototype with detailed view of the beam coupling unit (laser and measurement beam coupling).

the coupling system. In order to warrant a robust system with little long time deviations, e.g. by component wear, and little energy losses an optical element with a transmission of near to $100 \%$ needs to be chosen.

Another important system requirement is a highly accurate beam alignment. A misalignment between laser and measurement beam will lead to a displacement between the laser and the measurement spot, causing a mismatch / uncertainty in the measurement results.

The developed coupling system meets the described demands and enables a system integration by an unique machine hardware change. The insertion of a coupling optical element in the laser beam path with a determined angle $\left(45^{\circ}\right.$ for a dichroic beam splitter or a wavelength dependent angle for an optical filter) fulfills the complete integration. The component disposal for the beam coupling can be seen in the prototype setup presented in Figure 4.

The coupling efficiency of the concept was evaluated using an optical edge filter for the wavelength of $1064 \mathrm{~nm}$. By changing the angle of the edge filter, the edge frequency between reflection and transmission is displaced. By an angle of $23^{\circ}$ the edge frequency is adjusted in such a way, that the filter reflects the wavelength bandwidth of the measuring system and transmits the wavelength of the laser beam (Figure 4). An overall coupling efficiency of over $95 \%$ for the laser beam and over $93 \%$ for the measurement beam was evaluated in laboratory tests. These results validate the concept for the machine integration.

\subsection{Telecentric f-theta scanning lens}

As presented in Figure 1 a typical scanning system used in laser structuring systems is composed mostly of a scanning unit based on galvanometric moved mirrors and an f-theta scanning lens. The scanning objective used in the presented system prototype is a telecentric f-theta scanning lens. This lens type is wavelength optimized through the addition of a targeted optical distortion in the lens system. The aim of this optimization is to create a focal plane for the laser beam, as well as to create a direct proportional relation between scanning angle and laser spot position [4].

The designed optical system of a telecentric f-theta lens causes optical aberration in wavelengths other than the machining laser wavelength. This aberration introduces systematic errors to the measurement beam, such as a 


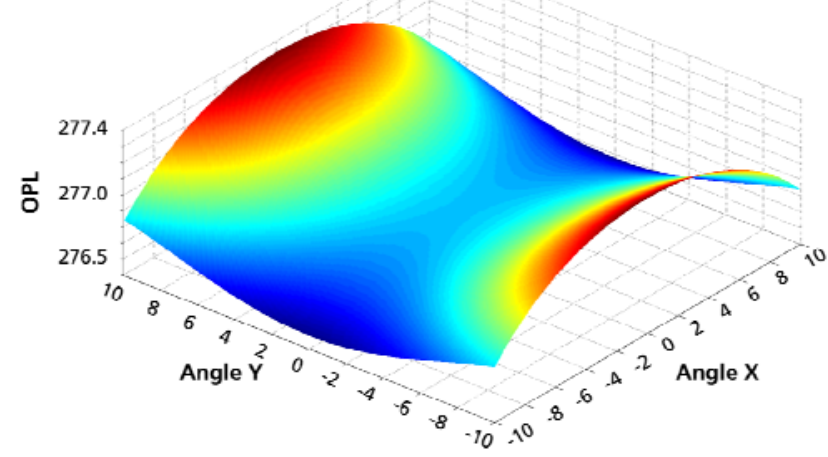

Figure 5. Simulation of the optical path length of the measurement beam through the machine optical system over the complete working field.

distortion of the focal plane, changes in the optical path and form deformation of the measurement spot. The evaluated dispersion could be compensated on the measurement system by the usage of a special designed glass rod, which was inserted on the system's reference path (Figure 1). This glass rod represents the same optical dispersion generated by the f-theta in the main optical path. Hereby the interference conditions are fulfilled for all field angles of the scanner.

The remaining effects of the f-theta in the optical path for other beam entrance angles can be seen in the measurement results as slight deviations of the real object form or a small decrease of the lateral accuracy in the border of the scanning field. The specific optical path deformation over the scanning field could be evaluated through a system simulation. The results show a form error in the shape of a saddle (Figure 5).

\subsection{Laser structuring system Prototype}

In order to evaluate the proposed inline measurement technique previously to a machine integration, a laser structuring system prototype was constructed. For the deflection of the laser and measurement beam a galvanometer based scanning unit was used.

A telecentric f-theta lens with focal distance of $80 \mathrm{~mm}$ was applied for focusing both beams on the structuring plane (Figure 6). The combination of both optical elements enables a working field of $30 \mathrm{~mm} \times 30 \mathrm{~mm}$. As machining laser a nanosecond pulsed fiber laser with central wavelength at 1064 $\mathrm{nm}$ was used. The controlling of the scanner and laser units is executed by a specially developed piece of software. A laser security compliant housing completes the prototype.

\section{RESULTS}

The evaluation of the system prototype was carried out through a series of test measurements in flatness and step standards, as well as in laser structured workpieces. For these tests the processing laser was turned off.

By measuring a flatness and a step standard the remaining amount of optical aberration introduced by the telecentric f-theta lens could be investigated. Alterations in the optical path length of the measuring beam affect the surface form inspection and need to be characterized.

The measurement of a flatness standard shows a slight distorted plane (Figure 7), as expected by the system simulation results (Figure 5). For a measurement area of $20 \mathrm{~mm} \times 20 \mathrm{~mm}$ a parabolic distortion could be detected in $x$

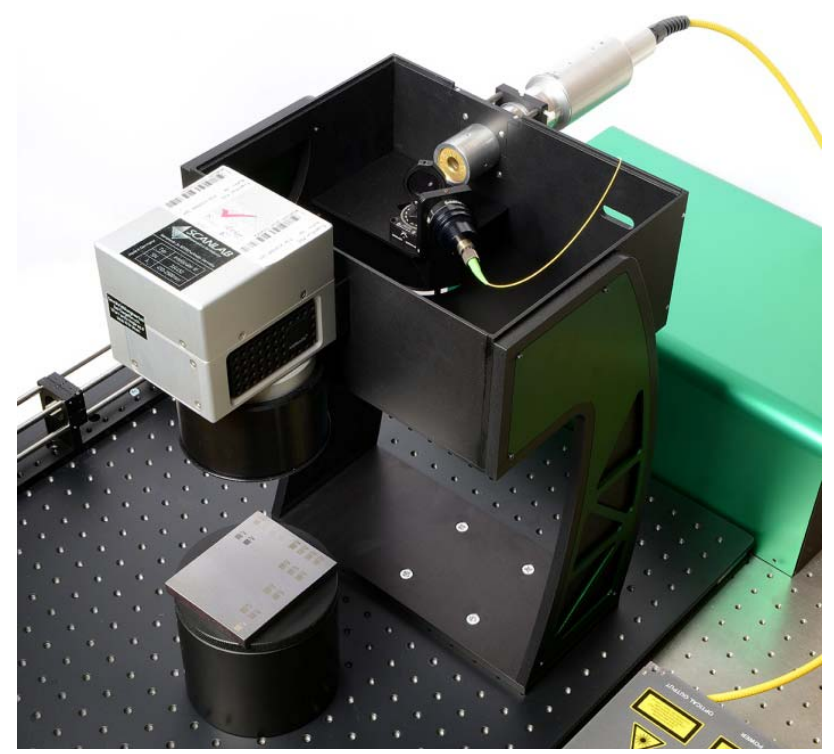

Figure 6. Prototype of a laser structuring system with the developed inline measurement system.

and $y$ directions. After the subtraction of the plane inclination a maximal deformation of $48 \mu \mathrm{m}$ in the $x$-axis and of $65 \mu \mathrm{m}$ in the $y$-axis was measured. As already shown in the system simulation, this measurement distortion is caused by an optical dispersion in dependency of the beam entrance angle.

A correction of this effect can be achieved by a system calibration, which is based on a measurement of a reference surface (e.g. flatness standard). The evaluated form error is modeled by a 3D polynomial and used in the system software to compensate the measurement results.

To evaluate the measurement range as well as a possible non-linearity after the calibration process a step standard with steps of about $100 \mu \mathrm{m}$ was measured (Figure 8 ). The measured area of the workpiece was $6.5 \mathrm{~mm} \times 2 \mathrm{~mm}$. The overall height variation measured by the prototype was $925 \mu \mathrm{m}$ over the 10 steps. A reference measurement with a chromatic sensor acquired a height variation of $917 \mu \mathrm{m}$ over the 10 steps. An overall non-linearity of about $8 \mu \mathrm{m}$ over a measurement range of $1 \mathrm{~mm}$ could be evaluated. This represents a non-linearity of

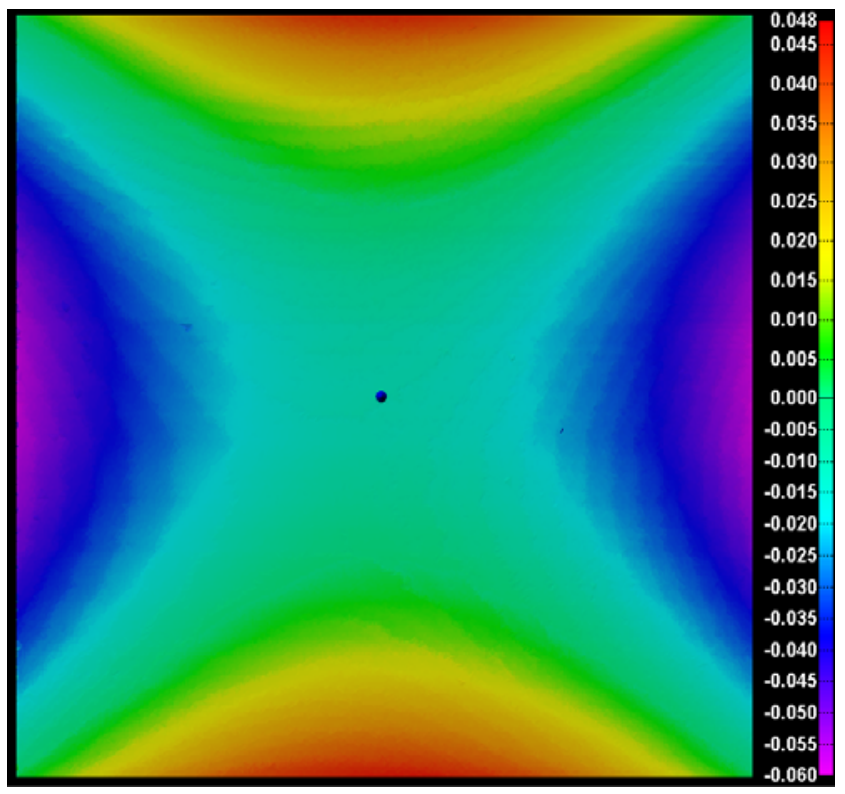

Figure 7. 3D Measurement of a reference surface (unit in $\mathrm{mm}$ ). 


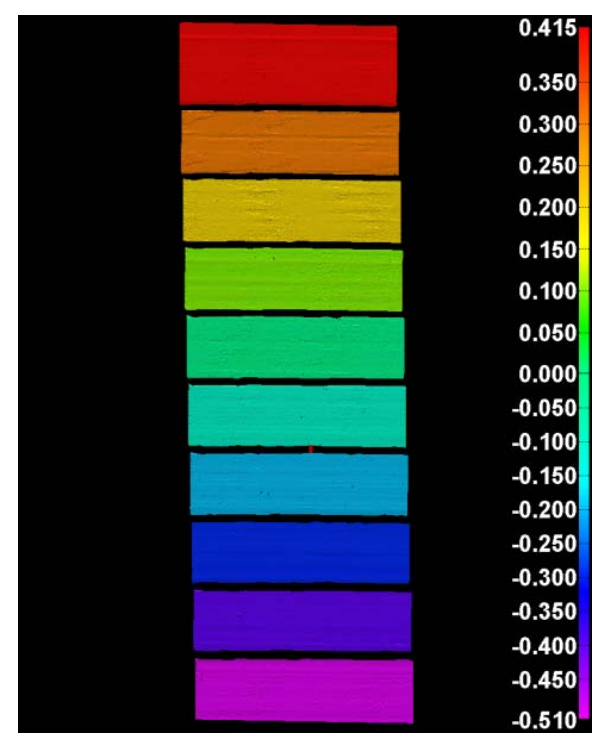

Figure 8. 3D Measurement of a step standard (unit in $\mathrm{mm}$ ).

less than $1 \%$ for the presented prototype in the used scanning area.

Regarding the application of the presented measurement system for laser structuring processes, e.g. in the manufacturing of tools and moulds [1], a laser machined structure $(20 \mathrm{~mm} \times 20 \mathrm{~mm}$ ) was measured and analyzed (Figure 9). The resulting measured surface demonstrates the robustness of the inline system for the inspection of complex surfaces.

\section{CONCLUSIONS}

Within this paper an optical measurement system for the surface inspection of micro and macro structures with sub-micron accuracy within a laser structuring machine was described and evaluated. A standard deviation of the distance measurements in the z-axis of fewer than $218 \mathrm{~nm}$ and a nonlinearity of less than $1 \%$ for a measurement range of $1 \mathrm{~mm}$ was determined.

Measurements on standards and laser structured surfaces are presented, which validate the potential of this technique for a telecentric surface inspection within laser structuring machines.

Future investigations, especially in the usage of this technique for a feedback control of adaptive laser micro processing machines, will be carried out. The effects of process emissions in the measurement results are also an important research task.

\section{ACKNOWLEDGEMENT}

We gratefully acknowledge the financial support by the German Ministry of Education for the project 'Scan4Surf' (02PO2861), which is the basis for the proposed achievements.

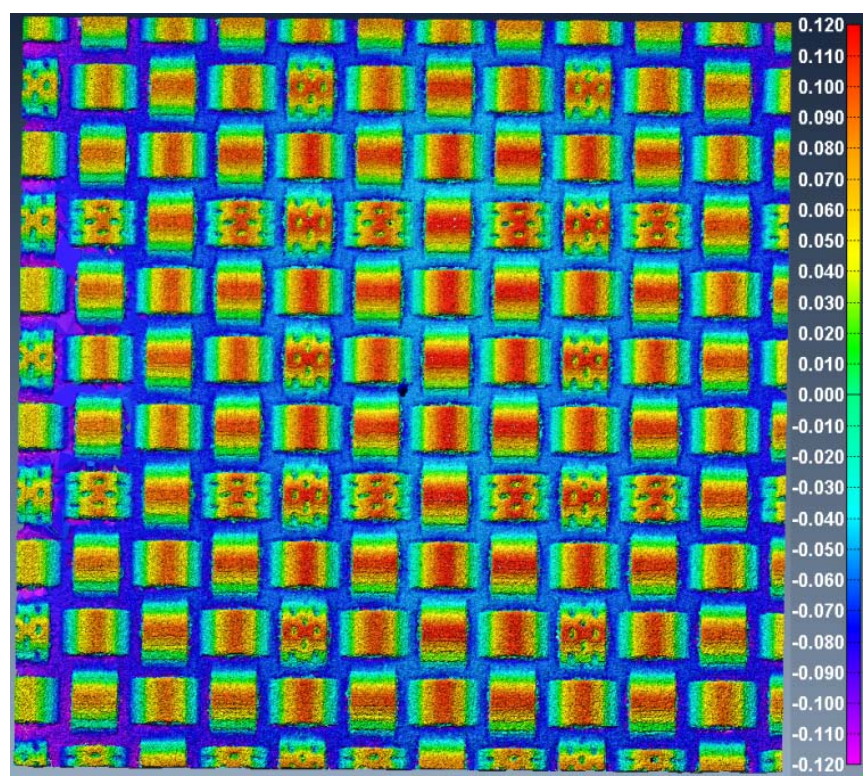

Figure 9. 3D Measurement of a laser structured surface (unit in $\mathrm{mm}$ ).

\section{REFERENCES}

[1] S. Schreck et. al., "Laser-assisted structuring of ceramic and steel surfaces for improving tribological properties", Proc. of the European Materials Research Society, Applied Surface Science, 2005, vol. 247, pp. 616-622.

[2] F. Klocke, et al., "Reproduzierbare Designoberflächen im Werkzeugbau: Laserstrahlstrukturieren als alternatives Fertigungsverfahren zur Oberflächenstrukturierung", Werkstatttechnik, 2009, no. 11/12, pp. 844-850.

[3] J. C. Ion, Laser Processing of Engineering Materials principles, procedure and industrial application, Elsevier, 2005, ISBN 978-0-7506-6079-2, p. 389.

[4] B. Furlong and S. Motakef, "Scanning lenses and systems", Photonik international, no. 2, 2008, pp. 20-23.

[5] T. Craeghs et. al., "Online Quality Control of Selective Laser Melting”, Proceedings of the 20th Solid Freeform Fabrication (SFF) symposium, 2011.

[6] M. Brezinski, Optical coherence tomography - Principles and Applications, Elsevier, 2006, ISBN 978-0121335700 pp. $130-134$.

[7] P. Tomlings and R. Wang, "Theory, developments and applications of optical coherence tomography", Applied Physics, no. 38, 2005, pp. 2519-2535.

[8] A. Rogalski, Infrared Detectors, CRC Press, 2010, ISBN 978-1420076714, pp. 315-317.

[9] R. Schmitt, G. Mallmann and P. Peterka, "Development of a FD-OCT for the inline process metrology in laser structuring systems”, Proc. SPIE 8082, 2011, 808228. 